\title{
Several Dots-to-Dot Audiovisual Flowing Above Parallel-to-parallel Networks
}

\author{
Mohammed Jasim Mohammed \\ College of Engineering, Nawroz University, Duhok, Kurdistan Region - Iraq
}

\begin{abstract}
TCP is that the transport protocol used copious larger in vary among the net. Protocol could also be a sender-driven protocol throughout that the sender collects knowledge to figure out the routing path. Specifically, we have a tendency to tend to inspire transport layer support for multipoint-to-point connections to wear down the matter of sources lacking properties like capability and handiness in Parallel-to-parallel networks. Here, we have a tendency to tend to require under consideration the matter of Several Dot-to-Dot Audiovisual Flowing Above Parallel-to-parallel networks. we have a tendency to tend to gift a convey category protocol spoken as R2CP (Beam Levee Rolling Protocol) that permits Several Dot-to-Dot Audiovisual Flowing Above Parallel-to-parallel Networks.R2CP could also be a receiver induces divers event convey protocol. It wants no coordination between multiple sources. It uses TCP-genial crowding management then sends to the Audiovisual Flowing the mix those bandwidths available on individual ways.
\end{abstract}

KEYWORDS: Several Dots-to-Dot, TCP-genial crowding, Parallel-to-parallel Networks.

\section{INTRODUCTION}

In the previous few years the Parallel-to-parallel Networks space has appealed abundant attention among the user. info and resources shared within the peer-to-peer network ar distributed within the localized manner, and recoverable on to any host taking part within the network. Present researches add the realm of peer-to-peer networking and have chiefly targeted on application layer approaches with vertical integrated solutions. To facilitate the event and ability of peer-to-peer applications, the growing scale and variety of peer-to-peer networks have entailed a typical

Academic Journal of Nawroz University

(AJNU) Volume 7, No 3 (2018).

Received 3 March 2018;

Regular research paper : Published 20 June 2018

Corresponding author's e-mail : Mjm562000@yahoo.com

Copyright (C2018 Mohammed Jasim Mohammed.

This is an open access article distributed under the

Creative Commons Attribution License. platform. Several of the researches tried to create generic architectures, interfaces and protocols that may support the peer-to-peer networks a lot of with efficiency and effectively. Transmission management Protocol (TCP) has been thought of because the largely used transport protocol within the net world and additionally within the peer-to-peer networks. The aforementioned network has designed for a unicast association between a server and a shopper and it exhibit numerous characteristics from those of typical client-server networks. Attributable to their restricted capability and transient handiness, these peers networks that act as sources to produce the content usually don't exhibit server like properties. While many approaches projected for period of stage flowing in content supply networks leverage the presence of several include servers [5, 6], this results cannot be used for Parallel-to-parallel Networks. One attention-grabbing communication paradigm that has been used with Parallel-to-parallel Networks is Several Dot-to-Dot communications. One consumer (requesting peer) will use several servers (supplying peers) to admission the required gratified, and improvement from 
the ensuing parallelization of the admission. The applications like Gaza area unit restricted to un factual period of loading and can't be apply for period of time flowing, although the very reality that the foremost well-liked style of records mutual in Parallel-to-parallel networks is transmission information's like music and video [1]. during this effort, we tend to specialize in the matter of enabling period of Audiovisual Flowing above Parallel-to-parallel victimization Several Dot-toDot contacts. The aim is to permit consumers to beginning out looking at the audiovisual once a brief delay (a small number of moments), while not necessity to attend (aimed at probably intervals) till the information is totally moved on the native disk. The applications of Parallel-to-parallel has up to now been restricted to un factual folder downloads. The applications wont to transfer any files has to be utterly downloaded on the native disk before it may be used. In use after-download mode, an extended delay is encountered. Such an extended waiting delay may be evaded if the consumer is given the flexibility toward audiovisual stream, whereas downloading. During this article, we have a tendency to aim to modify such period. Traditional approaches that open one unicast affiliation between the streaming server and also the shopper therefore can fail to produce users in Parallelto-parallel Networks with the flexibility to playback whereas downloading. Withal such borders, apparently the Parallel-to-parallel grid is additionally be distinguished by a great amount of information duplication thanks to separate Parallel performing as each shoppers and servers. For any content question, existing Parallel-to-parallel operation rules will expeditiously find many peers with the specified content. Therefore, during this article we have a tendency to target an answer wherever the demanding peer uses a Several Dot-to-Dot affiliation, as shown in Fig.1, to at the same time following the information from several supply peers for realizing the specified operation rate at the receiver.

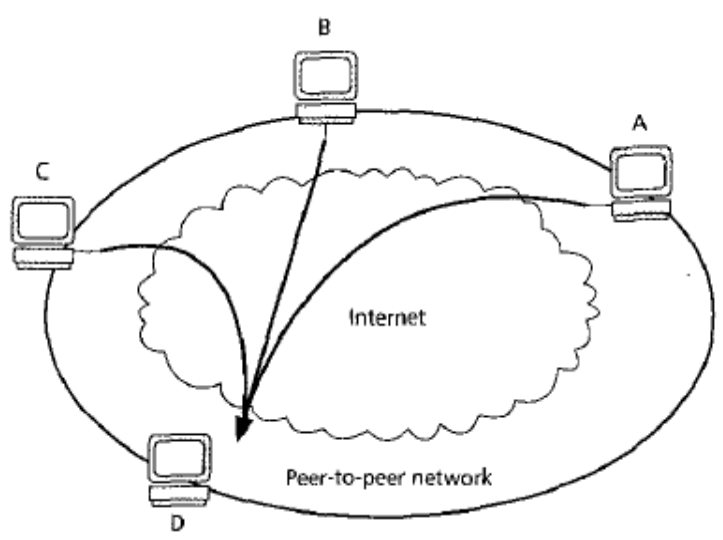

Figure (1) : .Multipoint-to-point communication

The advantage of this system is that the facility to combination the bandwidths out there on several methods from separate foundations to the destination. The 2 key dares concerned in completing multipoint-topoint video streaming over peer-to-peer networks are peer heterogeneousness and peer transience.

\section{MATERIALS AND METHODOLOGY}

\subsection{The $\mathbf{R}^{2} \mathrm{CP}$ Protocol Impression}

$R^{2} C P$ is a receiver-motivated, multistate passage procedure that cares together unicast and multipoint-topoint connection.

For instance shown in the following figure the assembly with $\mathrm{k}$ bases can be decomposed into the next elements.

- $\mathrm{k}$ RCP pipes that join separable transmitters to receiver

- $A R^{2} C P$ engine that matches several $R C P$ tubes at the receiver

The four key design elements in a multipoint-to-point transport protocol are: receiver-driven operation, maintaining multiple states, decoupling of functionalities through dynamic binding and packet scheduling. 


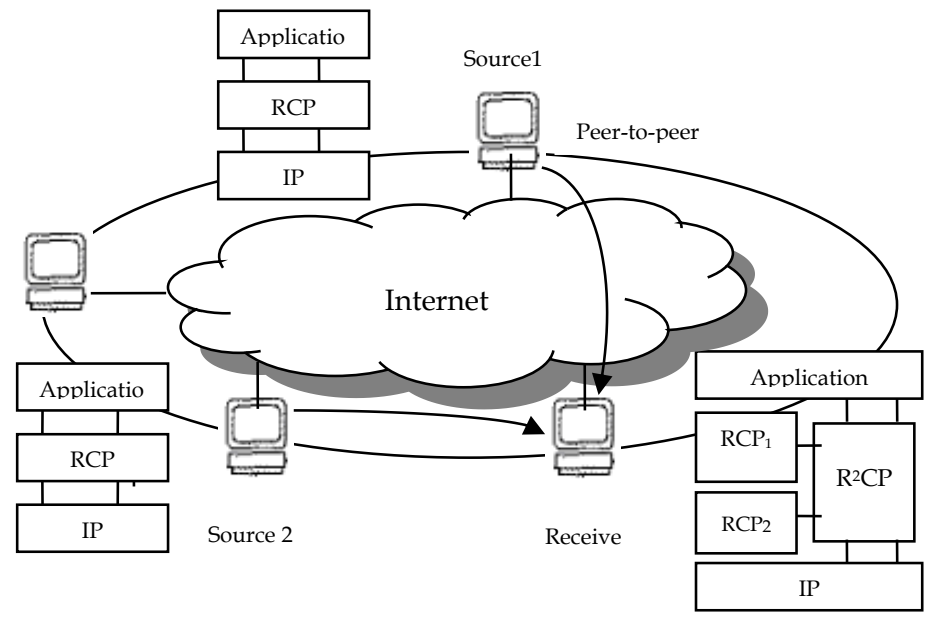

Figure (2) : R2CP for multipoint-to-point announcement

\section{Receiver driven operation}

The device be located in the accepter. RCP is a TCP clone that reuses most of the transport mechanisms in $\mathrm{TCP}$, consist a space-founded congestion device apparatus such as binomial crowding control [11].RCP uses request-data protocol handshake. The receiver in $\mathrm{R}^{2} \mathrm{CP}$ connection includes functionalities such as jamming device, damage recovery and packet arrangement. $\mathrm{R}^{2} \mathrm{CP}$ decouples the rules functionalities attendant with separate routes.

\section{Maintaining multiple states}

The R2CP destination maintains multiple states; every of them correspond to the one state maintained by individual sources within the association. $\mathrm{R} 2 \mathrm{CP}$ minimizes the overheads thanks to maintaining multiple states.

Dynamic binding

$\mathrm{R} 2 \mathrm{CP}$ permits knowledge recovery to occur on the $\mathrm{RCP}$ pipe completely different from the one knowledge was sent. This is often achieved in R2CP mistreatment through dynamic binding of the appliance knowledge and therefore the RCP packets mistreatment the binding organization (see Fig 3). Effectively, individual RCPs management what quantity the info to request from each sender, while the $\mathrm{R}^{2} \mathrm{CP}$ engine controls which data to request from each sender.

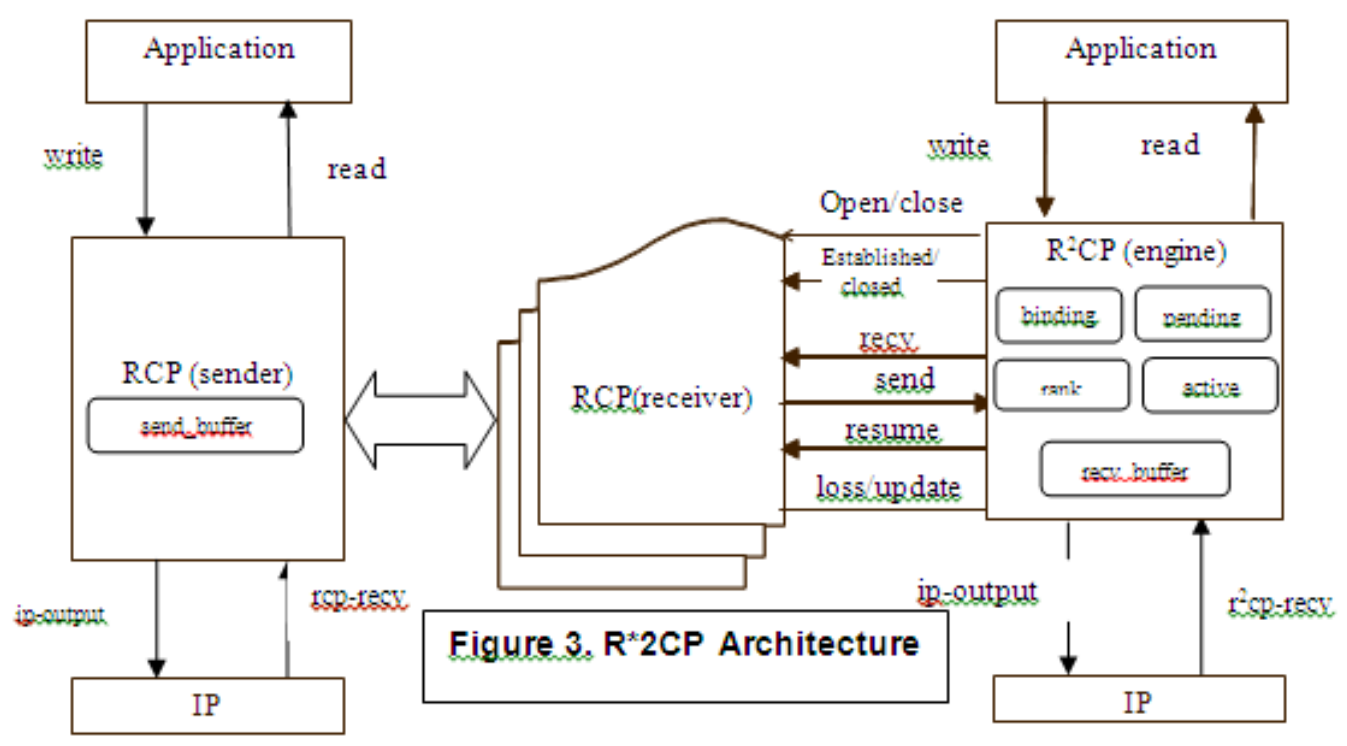


The $\mathrm{R}^{2} \mathrm{CP}$ engine at the receiver maintains the global sequence number, individual RCPs maintain limited structure figures for their procedure actions. Therefore, even though the congestion control mechanism used in $\mathrm{RCP}$ is designed for in-sequence delivery, the $\mathrm{R}^{2} \mathrm{CP}$ engine can retrieve non-contiguous data from each source. The mappings among the indigenous and worldwide structure numbers are maintained via $\mathrm{R}^{2} \mathrm{CP}$ device.

From the view of the $\mathrm{R}^{2} \mathrm{CP}$ device, the mandatory facts structure allows it to control which application data should be assigned to which RCP pipe depending on its transmission schedule.

\section{Packet Scheduling}

The $\mathrm{R} 2 \mathrm{CP}$ engine performs packet programing to coordinate packet transmissions on individual RCP pipes. R2CP uses associate RTT-ranked, CWND-based packet programing algorithmic program to reduce outof-order arrivals at the receiver. A key task that has to be performed by the $\mathrm{R} 2 \mathrm{CP}$ engine is to schedule requests of application information. the target is to reduce the amount of packet losses at the receiver as a results of either barrier surplus or deadline ending. The $\mathrm{R} 2 \mathrm{CP}$ engine uses the arrivals of knowledge to clock the transmissions of latest requests, and schedules missive of invitation on a path only the involved RCP pipe has house in its congestion window for requests. Whereas one easy thanks to schedule missive of invitation is to assign following world sequence variety to the new request, such first-come-first-served (FCFS) programing can cause out-of-order arrivals. The assignment instead ought to be supported the potential order of knowledge packets arrivals. The R2CP engine maintains a rank arrangement for locating the rank of the new request. Specifically, whenever the $\mathrm{R}^{2} \mathrm{CP}$ engine sends out a request for sequence number $\mathrm{i}$ through pipe $\mathrm{j}$ : an entry is added per a timestamp of $S=T_{i}+R_{T T}$, where $T$, is the period when the demand is sent, and RTT, is the round-trip period of pipe $\mathrm{j}$. The timestamp returns the period when new requests will be delivered in reply to the coming of the demanded information. As soon as the $\mathrm{R}^{2} \mathrm{CP}$ device obtains the send () request from pipe $\mathrm{k}$ at period $\mathrm{T}$, it localizes the rank $\mathrm{r}$ of the demand as

$$
r=1+\sum_{n=1}^{N}\left\lfloor\frac{T+R T T_{k}-S_{n}}{R T T_{p(n)}}\right\rfloor^{+}
$$

where $N$ is the overall amount of accesses in rank, $\mathrm{S}_{\mathrm{n}}$ is the timestamp at access $n, \mathrm{p}(\mathrm{n})$ is the pipe that directed out the demand at access $n$, and $\llcorner x\lrcorner^{+}$is the extreme of zero and the biggest digit fewer than or equivalent to $x$.

\subsection{How it works}

The functionalities achieved by $\mathrm{R} 2 \mathrm{CP}$ square measure affiliation management, congestion management, flow management, dependableness, packet programming and decoupling functionalities.

\section{Connection Management:}

When R2CP creates AN RCP pipe, it uses the open () decision to create the RCP pipe begins the association setup procedure. The setup method consists of constant acknowledgement as in transmission control protocol. But, when association is proven, rather than the dispatcher causing primary information section, the RCP recipient conveys the primary REQ with the tentative series variety. The dispatcher then conveys the primary information section at the time of delivery the REQ. The association rending in RCP conjointly abide by in transmission control protocol.

Once the RCP pipe is established, it uses the established () decision to advise R2CP. The R2CP association is established once any of the RCP pipe returns with the established () decision. On the opposite hand, once R2CP deletes AN RCP pipe, it uses the close() decision to create the RCP pipe enter the closing acknowledgement. once all RCP pipes come with the closed() decision, the R2CP association is closed.

\section{Congestion Control}

Congestion management in AN R2CP affiliation is operated on a per pipe basis, wherever every RCP pipe is accountable for dominant the number of information transferred through the individual path. $\mathrm{R} 2 \mathrm{CP}$ decides the congestion management mechanism to use for every interface by gap AN applicable RCP pipe. We have a tendency to use binomial congestion management algorithmic program in order to avoid congestion

\section{Flow Control}

Since R2CP has management over the receive buffer, it's chargeable for the flow management of the combination affiliation. $\mathrm{R} 2 \mathrm{CP}$ restrictions a demanding $\mathrm{RCP}$ pipe if it finds that the amount of outstanding information is capable the accessible buffer house. It de-freezes involved pipes through the resume () decision once any house is formed within the buffer. In RCP, since the receiver maintains the receive buffer and has total management over what quantity information the sender will send, flow management is internal to the receiver.

\section{Reliability}

R2CP remains mainly accountable on behalf of the dependable knowledge relocation of the mixture association, Accomplishes this objective via keeping the tying data aimed at totally knowledge sections. When a section is absolute toward a specific RCP cylinder, the involved cylinder in charge of. However, note that once Associate in Nursing RCP pipe detects a section loss via 3 out-of-order arrivals or timeouts [7], and reports to $\mathrm{R} 2 \mathrm{CP}$ victimization the loss() decision, $\mathrm{R} 2 \mathrm{CP}$ can detach the corresponding knowledge section, and give the trustworthy removal of the vanished section to subsequent offered cylinder. whereas the initial RCP pipe can still attempt to deliver constant section 
(conditions of the series range of RCP) by resend, it'll be allotted a numerous knowledge section via R2CP.If the RCP pipe incorporates a contemporary guesstimate for $\mathrm{RTT}$, it usages the update() request to report the $\mathrm{R} 2 \mathrm{CP}$ engine. While (p`s) interval in its crowding space has the direct () call (with local structure integer $l$ as the parameter) to request for broadcast at time localizes the rank $r$ of the demand using the rank data construction. The $\mathrm{r}^{\text {th }}$ data to request in the incomplete information construction is recognized as the section with sequence numbers. The $\mathrm{R}^{2} \mathrm{CP}$ device then produces a placeholder in recv-buffer supposing the coming of information sending. It enhances for data $g$ an entry $(p, l)$ in the tie information construction. Lastly sends out the request packet to the IP layer using as the arrangement number and then eliminates $g$ from the awaiting information construction. When the demand package reaches at the directing end of duct $p$, the sender finds in its drivebarrier the information with arrangement numbers, and guides out the information package to the IP layer. The transmitter then purgatives information in its barrier with structure numbers lower than $h$. Then the transmitter purely repeats whatnot information is requested from the destination, out-of-control conductions are probable at separable transmitter. When information fragment $g$ reaches, the $\mathrm{R}^{2} \mathrm{CP}$, device enqueuer the information to its representative in recvbuffer, discovers the corresponding RCP tube $\mathrm{p}$ and the limited sequence number $l$ founded on the tie information construction, and permits $l$ for concerned RCP tupe using the recv() call. It then removes the matching accesses in the tie and rank information structures. The concerned RCP pipe updates its states, limits either it can transmit additional demands or else based on the size of its congestion window. If it can create additional demands, it uses the send () call per the following local series figure for conduction demand such as earlier.

\section{COMPARISON WITH EXISTING SYSTEM}

The Protocols TCP with UDP are designed for point-topoint communication. It is not suited for streaming application because its reliability and ordering semantics increases end-to-end delay and delay variation. TCP is sender -centric protocol since sender performs congestion control and reliability. Also, TCP is not a scalable option for geographically distributed sources. TCP is a data-acknowledgement protocol. RCP is a TCP clone that reuses transport mechanisms in TCP. In RCP, the receiver drives the operation of the protocol. $R C P$ is a request-data protocol. $R^{2} C P$ is a multistate transport protocol that aggregates multiple $\mathrm{RCP}$ connections into one connection.

\section{Simulation Model:}

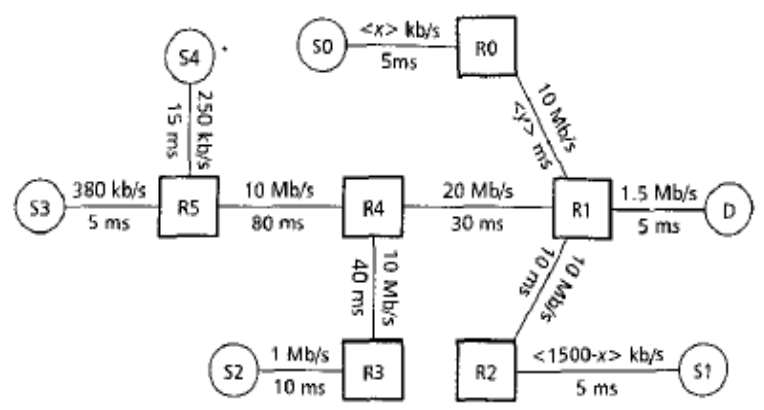

Figure 4. Network Topology

The network topology is founded on ns-2 net simulant. The linkage topology contains of (6) routers represented by square and (6) admittance nodules represented by circle connected via duplex contacts with bandwidth and transmission intervals as shown in fig .4.The figure shows the amount of data via route of Several Dot-to-Dot connection. As well as the objective multipoint-to-point joining, we introduce the next background traffic while the streaming takes place: We use a TCP stream S2 to S1 using FTP as the stream of traffic foundation. An on/off UDP stream from S3 to R0 using the Pareto traffic source using a mean eruption period of $1 \mathrm{~s}$, a mean lazy period of $2 \mathrm{~s}$, a data rate in the course of eruption period of 200 kbps. An on/off UDP flow from S4 to R4, with the following parameters: $0.5 \mathrm{~s}, 1 \mathrm{~s}$ and $100 \mathrm{kbps}$. Five TCP flows between R1 and R5 (bidirectional).On/off UDP streams between R1 and R5 (in both ways).All pipes in the multipoint-to-point connection use binomial crowding control [11] with the next factors: $\mathrm{k}=1.0,1=0$, $\alpha=1.0, \beta=0.66$. We use TCP/Sack for all TCP flows.

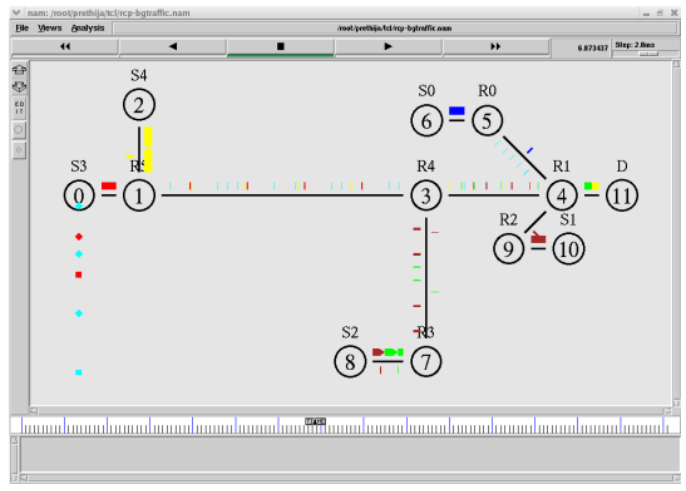

Figure (5) : TCP flow with UDP as background traffic

Fig .5 shows TCP flow with UDP as background traffic. We fixed the bandwidth of link S0-R0 to $128 \mathrm{~kb} / \mathrm{s}$ and delay of link R0-R1 to $20 \mathrm{~ms}$. 


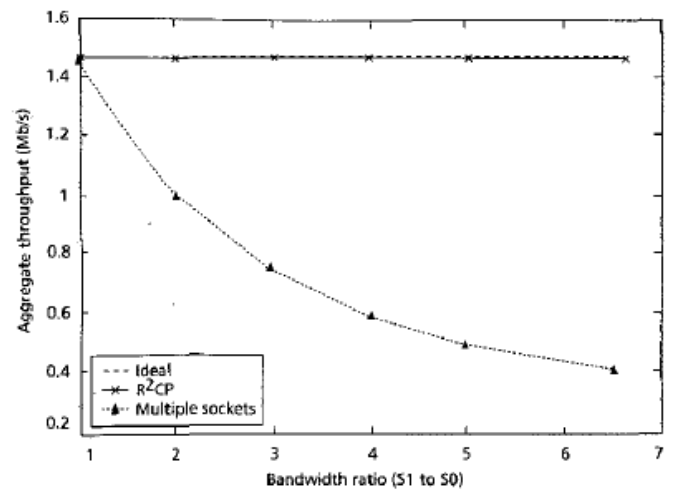

Figure (6) : Scalability with peer heterogeneity

\section{CONCLUSION}

In this article we investigate the problem Several Dot-toDot Audiovisual Flowing Above Parallel-to-parallel Networks. We present a carrying layer procedure called $\mathrm{R}^{2} \mathrm{CP}$ that efficiently allows actual-period Several Dot-toDot Audiovisual Flowing from diverse peers showing large bandwidth and postponement misalliances along individual end-to-end paths. $\mathrm{R}^{2} \mathrm{CP}$ is a receiver-driven, without management between multiple sources, provides accommodations supple placement category dependability semantics, and uses TCP-welcoming crowding monitor.

\section{REFERENCES}

1.S.Saroiu, P.Gummadi, and S.Gribble, (2002), "A Measurement Study of Peer-to-Peer File Sharing Systems, "Proc. SPIEMMCN, San Jose, CA, Jan, 2002.

2.P.Rodriguez and E.Biersack. , (2002),"Dynamic parallel access to replicated content in the internet" IEEE/ACM Transactions on Networking, vol.10, no. 4, Aug 2002 pp, 455-465.

3.John W. Byers, Michael Luby, Michael Mitzenmacher,(1999), "Accessing multiple mirror sites in parallel: Using tornado codes to speed up downloads", IEEE INFOCOM 1999 - The Conference on Computer Communications, no. 1, March 1999 pp, 275-283.

4.H.Y.Hsiah and R.Sivakumar, (2002), "A Transport Layer Approach for Achieving Aggregate Bandwidths on Multi-Homed Hosts", Proc.ACM MOBICOMM, Atlanta, GA, Sept, 2002.

5.John Apostolopoulos, Tina Wong, Wai-tian Tan, Susie Wee,(2002), "On multiple description streaming with content delivery networks", IEEE INFOCOM 2002 - The Conference on Computer Communications, vol. 21, no. 1, June 2002 pp, 1736-1745.

6.T.Nguyen and A.Zakhor,(2002),"Distributed Video Streaming Over Internet", Proc. SPIE MMCN, San Jose, CA, Jan, 2002.

7.H.Y.Hsieh et al, (2003),"A receiver-centric Transport Protocol for Mobile Hosts with Heterogeneous Wireless Interfaces",Proc. ACM MOBICOM,San Diego,CA,Sep, 2003.

8.S.Sen and J.Wang, (2002), "Analyzing Peer-to-Peer Traffic Across Large Networks,"Proc. ACM Internet Measurement Wksp.,Marseille,France,Nov ,2002.

9.D.Xu et al, (2002),"On peer-to-peer Media Streaming", Proc. IEEE ICDCS, Vienna, Austria, July, 2002.

10. M.Hefeeda et al, (2003),"PROMISE: Peer-to-Peer Media Streaming Using Collect Cast", Proc.ACM Multimedia, Berkeley, CA, and Nov ,2003.

11. Deepak Bansal, Hari BalaKrishnan, (2001), "Binomial congestion control algorithms", IEEE INFOCOM 2001 The Conference on Computer Communications, no. 1, April 2001 pp. 631-640

12.D.Clark and D.Tennenhouse, (2002),"Architectural Consideration for a new Generation of Protocols",Proc. ACM SIGCOMM ,Philadelphia,PA,Apr , 2002. 